\title{
In silico vs in vitro analysis of primer specificity for the detection of Gardnerella vaginalis, Atopobium vaginae and Lactobacillus spp.
}

\author{
Ana Henriques, Tatiana Cereija, António Machado and Nuno Cerca*
}

\begin{abstract}
Background: Bacterial vaginosis (BV) is a common pathology of women in reproductive age that can lead to serious health complications, and is associated with shifts in the normal microflora from predominance of Lactobacillus spp. to a proliferation of other anaerobes such as G. vaginalis and A vaginae, which can be detected by PCR. The optimal PCR pathogen detection assay relies mainly on the specificity and sensitivity of the primers used.

Findings: Here we demonstrate that in silico analytical testing of primer specificity is not a synonym to in vitro analytical specificity by testing a range of published and newly designed primers with both techniques for the detection of BV-associated microorganisms.

Conclusions: By testing primer in vitro specificity with a sufficient range of bacterial strains, we were able to design primers with higher specificity and sensitivity. Also by comparing the results obtained for the newly designed primers with other previously published primers, we confirmed that in silico analysis is not sufficient to predict in vitro specificity. As such care must be taken when choosing the primers for a detection assay.
\end{abstract}

Keywords: Primer specificity, Primer sensitivity, In silico primer testing, In vitro primer testing, G. vaginalis, A. vaginae, Lactobacillus spp.

\section{Findings}

Bacterial vaginosis (BV) is one of the most common vaginal disorders of women in reproductive age and is reported to be linked to increased risks of pre-term labor, HIV infection, postoperative infection and inflammatory pelvic disease [1]. It has been recognized that this pathology is caused by a shift in the microbial ecosystem colonizing the vagina of healthy women; from a Lactobacillus spp. dominated microbial population to the proliferation of other anaerobic microorganisms such as Gardnerella vaginalis, Atopobium vaginae, Mobiluncus spp. among others [2-4]. The etiology of BV remains unknown but a recent study reported that microbial biofilms, complex tridimensional structures that are known to be highly resistant to antimicrobial chemotherapy [5], may play a fundamental role in BV [6]. It has been described that G. vaginalis may account for 60 to $90 \%$ of the BV biofilm mass, while A. vaginae may

\footnotetext{
* Correspondence: nunocerca@ceb.uminho.pt

CEB-IBB, Centro de Engenharia Biológica - Instituto de Biotecnologia e Bioengenharia, Universidade do Minho, Campus de Gualtar, 4710-057, Braga, Portugal
}

account for 1 to $40 \%$ and Lactobacillus spp. for 1 to 5\% [7]. While trying to confirm these findings, in biofilms from vaginal samples of Portuguese women, using a quantitative PCR approach, we came across some difficulties related with primer specificity, despite the increase in the number of published papers regarding the use of molecular tools, such as PCR, for the detection of BV-associated microorganism [8-11]. In order to assess the usefulness of the primers for the detection of our target microorganisms used we decided to test the analytical specificity (\% of non-target organisms detected) and sensitivity (\% of target organisms detected) of the primers used. Being a quick and inexpensive method, in silico analysis of primer specificity and sensitibity has been widely used. However this technique poses some limitations, already described for other applications, namelly significant differences between the in silico prediction for primer specificity/sensitivity and the actual in vitro results $[8,9]$.

To our knowledge the analytical comparison of primer in silico and in vitro specificity/sensitivity for primers designed for the detection of BV-associated microorganisms has not 
been studied and with the increase in the use of molecular techniques for the study of BV such information becomes relevant.

We began by selecting primers previously described in the literature specific for Lactobacillus spp. [12,13], G. vaginalis $[8,14]$ and $A$. vaginae $[11,15]$. The reported primers specificity was mainly determined by in silico analysis using sequence alignment such as BLAST [16]. Whenever in vitro specificity was reported, often published details of such specificity determinations were scarce. When confirming primer specificity, using a few collection strains, and the same conditions reported in the literature, we found that some in silico primer specificity did not correspond to the in vitro specificity (data not shown). To address this, we selected the best primers that we could find in the literature and repeated the in silico specificity analysis using ProbeMatch [17]. We also designed new primers (using VectorNTI, version 11.0 and sequences available in the GeneBank databases) (Table 1).

During in silico analysis of the selected primers, we found two main problems: (i) the selected published primers for G. vaginalis were designed for the $16 \mathrm{~S}$ rRNAencoding DNA and 23S rRNA-encoding DNA intergenic region $[8,14]$ and there are no available databases which include this region that could be used for the in silico analysis; (ii) some of the primers for A. vaginae and Lactobacillus spp. showed low sensitivity in silico. In order to confirm our in silico analysis, in vitro testing of primer specificity was performed, initially with 3 strains of each target (G.vaginalis, A. vaginae and Lactobacillus spp., details in Additional file 1: Table S1), using DyNAnzyme PCR Master Mix 2x (Finnenzymes, Thermo Scientific, Finland). The initial amplification conditions comprised 40 cycles with the following temperatures each cycle: $94^{\circ} \mathrm{C}$ for 30 seconds, $60^{\circ} \mathrm{C}$ for 30 seconds, and $72^{\circ} \mathrm{C}$ for 1 minute. Amplified products were analysed in a $1 \%$ agarose gel and stained with Midori Green nucleic acid dye (Nippon Genetics Europe $\mathrm{GmbH}$, Germany). Results of the PCR at $60^{\circ} \mathrm{C}$ showed low sensitivity and specificity for Lactobacillus spp. primers, and as such we decided to optimize this detection with the aim of obtaining a greater specificity. The annealing temperature was then adjusted to $62^{\circ} \mathrm{C}$, and the number of strains increased to a total of 12 target strains (per group) and up to 34 non-target strains (including Pseudomonas aeruginosa, Staphylococcus epidermidis, Streptococcus agalactiae, Enterococcus faecalis, Escherichia coli and Staphylococcus cohnii) (Table 2). We used different annealing temperatures, in order to observe the effect that this variation would have in primer sensitivity and specificity, which is

Table 1 In silico analysis of primer specificity of the primers used in this study

\begin{tabular}{|c|c|c|c|c|c|c|c|c|}
\hline Target & Probe & & Sequence $\left(5^{\prime} \rightarrow 3^{\prime}\right)$ & $\begin{array}{c}\text { No. of target } \\
\text { strains detected }^{\text {a }}\end{array}$ & $\begin{array}{c}\text { Sensitivity } \\
(\%)^{\mathbf{b}}\end{array}$ & $\begin{array}{l}\text { No. of non-target } \\
\text { strains detected }^{a}\end{array}$ & $\begin{array}{c}\text { Specificity } \\
(\%)^{b}\end{array}$ & Reference \\
\hline \multirow[t]{6}{*}{ G. vaginalis } & Gard154-454 & FW & CTCTTGGAAACGGGTGGTAA & 36 (from 40) & 90 & 1 (from 1032184) & 100 & This study \\
\hline & & $\mathrm{RV}$ & TTGCTCCCAATCAAAAGCGGT & 38 (from 40) & 95 & 94 (from 1032184) & 99,99 & \\
\hline & $\mathrm{GV} 1+3$ & $\mathrm{FW}$ & TTACTGGTGTATCACTGTAAGG & & & & & {$[15]$} \\
\hline & & $\mathrm{Rv}$ & CCGTCACAGGCTGAACAGT & & & & & \\
\hline & $\mathrm{GV} 1+2$ & $\mathrm{FW}$ & TCCTGTCTACCAAGGCATCC & & & & & {$[13]$} \\
\hline & & $\mathrm{RV}$ & CGTGTGATAACCGTCAGGTG & & & & & \\
\hline \multirow[t]{6}{*}{ A. vaginae } & Atop109-329 & $\mathrm{FW}$ & GAGTAACACGTGGGCAACCT & 457 (from 467) & 97,86 & 18390 (from 1031757) & 98,22 & This study \\
\hline & & $\mathrm{RV}$ & CCGTGTCTCAGTCCCAATCT & 449 (from 467) & 96,15 & 1356 (from 1031757) & 99,87 & \\
\hline & AtovagRT3 & $\mathrm{FW}$ & GGTGAAGCAGTGGAAACACT & 134 (from 467) & 28,69 & 0 (from 1031757) & 100 & {$[11]$} \\
\hline & & $\mathrm{RV}$ & ATTCGCTTCTGCTCGCGCA & 109 (from 467) & 23,34 & 2 (from 1031757) & 100 & \\
\hline & Atop167-587 & FW & GCGAATATGGGAAAGCTCCG & 117 (from 467) & 25,05 & 0 (from 1031757) & 100 & {$[10]$} \\
\hline & & $\mathrm{RV}$ & TCATGGCCCAGAAGACCGCC & 115 (from 467) & 24,63 & 0 (from 1031757) & 100 & \\
\hline \multirow{6}{*}{$\begin{array}{l}\text { Lactobacillus } \\
\text { spp. }\end{array}$} & AM Lacto & $\mathrm{FW}$ & TGATGCATAGCCGAGTTGAG & 7353 (from 12936) & 56,84 & 2513 (from 1019288) & 99,75 & This study \\
\hline & & $\mathrm{RV}$ & AGCCGAAACCCTTCTTCACT & 5858 (from 12936) & 45,28 & 1027 (from 1019288) & 99,9 & \\
\hline & New Lacto & FW & TGGAAACAGRTGCTAATACCG & 11680 (from 12936) & 90,29 & 7069 (from 1019288) & 99,31 & {$[16]$} \\
\hline & & $\mathrm{RV}$ & GTCCATTGTGGAAGATTCCC & 10113 (from 12936) & 78,18 & 3311 (from 1019288) & 99,68 & \\
\hline & $\mathrm{S} 21+\mathrm{A} 19$ & $\mathrm{FW}$ & TGCCTAATACATGCAAGTCGA & 9754 (from 12936) & 75,4 & 184796 (from 1019288) & 81,87 & {$[17]$} \\
\hline & & Rv & GTTTGGGCCGTGTCTCAGT & 10157 (from 12936) & 78,52 & 72659 (from 1019288) & 92,87 & \\
\hline
\end{tabular}

Empty boxes correspond to the primers that could not be analysed because there are no databases available to encompass the intergenic region between $16 \mathrm{~S}$ and 235 rRNA genes for the in silico analysis.

${ }^{a}$ Calculated using ProbeMatch (last accession, May 2012) with the following data set options: Strain - Both; Source - Both; Size - > 1200 bp; Quality - Both.

${ }^{\text {b}}$ Formula: Specificity $=(\mathrm{nts} / \mathrm{Tnts})^{*} 100$ (nts: number of non-target strains undetected, Tnts: total number of non-target strains tested). Sensitivity $=(\mathrm{ts} / \mathrm{Tts})^{*} 100$ (ts: number of target strains detected, Tts: total number of target strains tested). 
Table 2 In vitro analysis of primer specificity of the primers used in this study

\begin{tabular}{|c|c|c|c|c|c|c|c|}
\hline Target & Probe & $\begin{array}{l}\text { Annealing } \\
\text { temp }\left({ }^{\circ} \mathrm{C}\right)\end{array}$ & $\begin{array}{c}\text { No. of target } \\
\text { strains detected a }\end{array}$ & $\begin{array}{c}\text { Sensitivity } \\
(\%)^{\mathbf{b}}\end{array}$ & $\begin{array}{l}\text { No. of non-target } \\
\text { strains detected a }\end{array}$ & $\begin{array}{c}\text { Specificity } \\
(\%)^{\mathbf{b}}\end{array}$ & Reference \\
\hline \multirow[t]{6}{*}{ G. vaginalis } & Gard154-454 & 60 & 3 (from 3) & 100.0 & 0 (from 6) & 100.0 & This study \\
\hline & & 62 & 12 (from 12) & 100.0 & 0 (from 34) & 100.0 & \\
\hline & $\mathrm{GV} 1+3$ & 60 & 3(from 3) & 100.0 & 0 (from 6) & 100.0 & [15] \\
\hline & & 62 & 10 (from 12) & 83.3 & 0 (from 34) & 100.0 & \\
\hline & $\mathrm{GV} 1+2$ & 60 & 3 (from 3) & 100.0 & 0 (from 6) & 100.0 & [13] \\
\hline & & 62 & 11 (from 12) & 91.7 & 0 (from 34) & 100.0 & \\
\hline \multirow[t]{7}{*}{ A. vaginae } & Atop109-329 & 60 & 3 (from 3) & 100.0 & 5 (from 6) & 16.7 & This study \\
\hline & & 62 & 12 (from 12) & 100.0 & 15 (from 24) & 37.5 & \\
\hline & & 66 & 12 (from 12) & 100.0 & 3 (from 34) & 91.2 & \\
\hline & AtovagRT3 & 60 & 12 (from 12) & 100.0 & 1 (from 24) & 95.8 & [11] \\
\hline & & 62 & 12 (from 12) & 100.0 & 2 (from 34) & 94.1 & \\
\hline & Atop167-587 & 60 & 2 (from 3) & 66.7 & 0 (from 6) & 100.0 & [10] \\
\hline & & 62 & 11 (from 12) & 91.6 & 4 (from 34) & 88.3 & \\
\hline \multirow[t]{6}{*}{ Lactobacillus spp. } & AM Lacto & 60 & 2 (from 12) & 16.7 & 1 (from 24) & 95.8 & This study \\
\hline & & 58 & 2 (from 12) & 16.7 & 3 (from 34) & 91.2 & \\
\hline & New Lacto & 60 & 5 (from 12) & 41.7 & 18 (from 34) & 47,1 & [16] \\
\hline & & 62 & 1 (from 12) & 8.3 & 8 (from 24) & 66.7 & \\
\hline & $\mathrm{S} 21+\mathrm{A} 19$ & 60 & 5 (from 12) & 41.7 & 24 (from 24) & 0 & [17] \\
\hline & & 62 & 6 (from 12) & 50.0 & 34 (from 34) & 0 & \\
\hline
\end{tabular}

${ }^{a}$ Calculated using ProbeMatch (last accession, May 2012) with the following data set options: Strain - Both; Source - Both; Size - > 1200 bp; Quality - Both.

${ }^{b}$ Formula: Specificity $=(\mathrm{nts} / \mathrm{Tnts})^{*} 100$ (nts: number of non-target strains undetected, Tnts: total number of non-target strains tested). Sensitivity $=(\mathrm{ts} / \mathrm{Tts})^{*} 100$ (ts: number of target strains detected, Tts: total number of target strains tested).

information that could facilitate the use of these primers in combination (for multiplex PCR assays for example) for quick and effective PCR detection assay.

When comparing in silico predictions against in vitro results, we found considerable differences in specificity and sensitivity values. Despite the fact that theoretical melting temperature of the $A$. vaginae primers designed in this study was $60^{\circ} \mathrm{C}$, at this temperature primer specificity was very low. Only with higher annealing temperature $\left(66^{\circ} \mathrm{C}\right)$, we could achieve reasonable specificity (91.2\%) and sensitivity (100\%) values. The primers for Lactobacillus spp. were the ones with the lowest specificity. Of note, some of the primers previously reported as specific were found to be non-specific (0\%) despite an in silico prediction of $81 \%$ of specificity. A lower specificity was expected since these primers were designed for the identification of a genus, which results in a higher inherent genetic variability than primers for the identification a species. However, the accentuated decrease between in silico and in vitro primer specificity was not anticipated. The primers described by Pepin et al. (10) revealed the highest specificity for $A$. vaginae (Table 2), probably due to the previous in vitro specificity analysis against at least 20 isolates that was performed by the authors [10]. Although A. vaginae primers demonstrated an overall lower specificity, they proved to be highly sensitive, being able to detect all the targets used (Table 2). It is worth to note that the primer designed for this study for the detection of G. vaginalis proved to have a higher sensitivity than that of published primers, and that contrary to the results obtained for the other target microorganisms, the in silico prediction of sensitivity and specificity was similar to the in vitro results obtained.

It is interesting to note that in silico analysis for primers specificity was not a good predictor of in vitro primer specificity, something already reported by other researchers $[8,9]$, in silico predictions do not take into account the chemical reactions/limitations that can occur in the PCR tube and as such cannot truly approximate experimental events. In most articles describing the detection of BV-associated microorganisms the primer specificity testing is described as being performed in silico [10-15]. This work confirms the importance of proper in vitro analysis of primer specificity, as the in silico analysis can sometimes underestimate the possible cross reaction with non-sense strains or species which are not yet sequenced, as is the case of most of the strains present in vaginal environment. Furthermore, the new G. vaginalis and $A$. vaginae primers reported here have high in vitro specificity, demonstrating its potential for use in clinical microbiology. 


\section{Additional file}

Additional file 1: Table S1. Description of name and origin of strains used in this study for the in vitro testing of primer specificity and sensitivity.

\section{Competing interests}

The authors declare that they have no competing interests in this work. The funding organization did not play any role in the design of the experiments and article drafting.

\section{Authors' contributions}

This an original work that has not been published elsewhere. The experiments were designed by $\mathrm{NC}$ and $\mathrm{AH}$, the in vitro analysis was done by $\mathrm{AH}$ and $\mathrm{TC}$, primer design and in silico analysis was done by AM. AH drafted the manuscript, and all authors critically reviewed and agreed with the present manuscript.

\section{Acknowledgements}

This work was supported by European Union funds (FEDER/COMPETE) and by national funds (FCT) under the project with reference FCOMP-01-0124FEDER-008991 (PTDC/BIA-MIC/098228/2008). AM acknowledges the FCT individual fellowship SFRH/BD/62375/2009.

Received: 4 September 2012 Accepted: 14 November 2012

Published: 15 November 2012

\section{References}

1. Schmid GP: The epidemiology of bacterial vaginosis. Int J Gynaecol Obstet 1999, 67(Suppl 1):S17-S20.

2. Pirotta M, Fethers KA, Bradshaw CS: Bacterial vaginosis - more questions than answers. Aust Fam Physician 2009, 38:394-397.

3. Joesoeff MR, Schimd GP, Hillier SL: Bacterial vaginosis: review of treatment options and potential clinical indications for therapy. Clin Infect Dis 1999, 28:S57-S65.

4. Mastromarino P, Macchia S, Meggiorini L, Trinchieri V, Mosca L, Perluigi M, et al: Effectiveness of Lactobacillus-containing vaginal tablets in the treatment of symptomatic bacterial vaginosis. Clin Microbiol Infect 2009, 15:67-74.

5. Cos P, Tote $K$, Horemans T, Maes L: Biofilms: an extra hurdle for effective antimicrobial therapy. Curr Pharm Des 2010, 16:2279-2295.

6. Swidsinski A, Mendling W, Loening-Baucke V, Ladhoff A, Swidsinski S, Hale $L P$, et al: Adherent biofilms in bacterial vaginosis. Obstet Gynecol 2005, 106:1013-1023.

7. Srinivasan S, Fredricks DN: The human vaginal bacterial biota and bacterial vaginosis. Interdiscip Perspect Infect Dis 2008, 200(8):750479.

8. Huws SA, Edwards JE, Kim EJ, Scollan ND: Specificity and sensitivity of eubacterial primers utilized for molecular profiling of bacteria within complex microbial ecosystems. J Microbiol Methods 2007, 70:565-569.

9. Morales SE, Holben WE: Empirical testing of $16 \mathrm{~S}$ rRNA gene PCR primer pairs reveals variance in target specificity and efficacy not suggested by in silico analysis. Appl Environ Microbiol 2009, 75:2677-2683.

10. Pepin J, Deslandes S, Giroux G, Sobela F, Khonde N, Diakite S, et al: The complex vaginal flora of West African women with bacterial vaginosis. PLOS One 2011, 6:e25082.

11. De Backer E, Verhelst R, Verstraelen H, Alqumber MA, Burton JP, Tagg JR, et al: Quantitative determination by real-time PCR of four vaginal Lactobacillus species, Gardnerella vaginalis and Atopobium vaginae indicates an inverse relationship between $L$. gasseri and $L$. iners. BMC Microbiol 2007, 7:115

12. Ferris MJ, Masztal A, Martin DH: Use of species-directed 16S rRNA gene PCR primers for detection of Atopobium vaginae in patients with bacterial vaginosis. J Clin Microbiol 2004, 42:5892-5894.

13. McKechnie ML, Hillman R, Couldwell D, Kong F, Freedman E, Wang H, et al: Simultaneous identification of 14 genital microorganisms in urine by use of a multiplex PCR-based reverse line blot assay. J Clin Microbiol 2009, 47:1871-1877.

14. Menard JP, Fenollar F, Henry M, Bretelle F, Raoult D: Molecular quantification of Gardnerella vaginalis and Atopobium vaginae loads to predict bacterial vaginosis. Clin Infect Dis 2008, 47:33-43.
15. Zariffard MR, Saifuddin M, Sha BE, Spear GT: Detection of bacterial vaginosis-related organisms by real-time PCR for Lactobacilli, Gardnerella vaginalis and Mycoplasma hominis. FEMS Immunol Med Microbiol 2002, 34:277-281.

16. Byun R, Nadkarni MA, Chhour KL, Martin FE, Jacques NA, Hunter N: Quantitative analysis of diverse Lactobacillus species present in advanced dental caries. J Clin Microbiol 2004, 42:3128-3136.

17. Rekha R, Rizvi MA, Jaishree P: Designing and validation of genus-specific primers for human gut flora study. Electron J Biotechnol 2006, 9:505-5211.

doi:10.1186/1756-0500-5-637

Cite this article as: Henriques et al:: In silico vs in vitro analysis of primer specificity for the detection of Gardnerella vaginalis, Atopobium vaginae and Lactobacillus spp.. BMC Research Notes 2012 5:637.

\section{Submit your next manuscript to BioMed Central and take full advantage of:}

- Convenient online submission

- Thorough peer review

- No space constraints or color figure charges

- Immediate publication on acceptance

- Inclusion in PubMed, CAS, Scopus and Google Scholar

- Research which is freely available for redistribution 